\title{
Eigenvalues, absolute continuity and localizations for periodic unitary transition operators
}

\author{
Tatsuya Tate \\ Mathematical Institute, Graduate School of Sciences, \\ Tohoku University, Aoba, Sendai 980-8578, Japan \\ tatsuya.tate.c6@tohoku.ac.jp
}

Received 13 March 2017

Accepted 6 May 2019

Published 19 July 2019

Communicated by Luigi Accardi

\begin{abstract}
The localization phenomenon for periodic unitary transition operators on a Hilbert space consisting of square summable functions on an integer lattice with values in a finitedimensional Hilbert space, which is a generalization of the discrete-time quantum walks with constant coin matrices, is discussed. It is proved that a periodic unitary transition operator has an eigenvalue if and only if the corresponding unitary matrix-valued function on a torus has an eigenvalue which does not depend on the points on the torus. It is also proved that the continuous spectrum of a periodic unitary transition operator is absolutely continuous. As a result, it is shown that the localization happens if and only if there exists an eigenvalue, and when there exists only one eigenvalue, the long-time limit of transition probabilities coincides with the point-wise norm of the projection of the initial state to the eigenspace. The results can be applied to certain unitary operators on a Hilbert space on a covering graph, called a topological crystal, over a finite graph. An analytic perturbation theory for matrices in several complex variables is employed to show the result about absolute continuity for periodic unitary transition operators.
\end{abstract}

Keywords: Quantum walks; periodic unitary transition operators; localizations; absolutely continuous spectrum.

AMS Subject Classification: 35P05, 47A55, 58J50

\section{Introduction}

The term discrete-time quantum walks has been recently used to mean probability distributions, which is called transition probabilities, defined by using unitary operators. In this context, the unitary operators are often called unitary

This is an Open Access article published by World Scientific Publishing Company. It is distributed under the terms of the Creative Commons Attribution-NonCommercial-NoDerivatives 4.0 (CC BY-NC-ND) License which permits use, distribution and reproduction, provided that the original work is properly cited, the use is non-commercial and no modifications or adaptations are made. 
transition operators or unitary time-evolutions. Originally, the specific class of unitary operators in typical models are formulated in quantum physics ${ }^{2}$ and computer science. $1.3[20$ As in the theory of usual random walks, clarifying the asymptotic behavior, as time goes to infinity, of the transition probability is one of main issues for the investigation of quantum walks. It is well known that asymptotic behavior of quantum walks in typical models are quite different from that of usual random walks. The weak-limit distributions of quantum walks are usually ballistic, 17124 and the point-wise asymptotics are also quite different from that of the classical random walks. .23 One of the peculiar properties of quantum walks is a localization, which is a phenomenon that the transition probabilities, at some points, do not tend to zero as time goes to infinity. What is interesting is that, the localization phenomenon happens for quantum walks with very simple classes of unitary transition operators, even on the one-dimensional integer lattice, as discussed in Refs. 11, 12 and 13 There are numerous literatures on the localization phenomenon for quantum walks and it would be hard to mention them all, thus we just refer Ref. 18 where the localization properties of a quantum walk on certain infinite graphs called spidernets, not on the usual integer lattices, are discussed.

To be more concrete, let $Z$ be a countable set and let $W$ be a finite-dimensional Hilbert space with the inner product $\langle\cdot, \cdot\rangle_{W}$. Let $U$ be a unitary operator on the Hilbert space $\ell^{2}(Z, W)$ consisting of all square summable functions on $Z$ with values in $W$ with the inner product defined by

$$
\langle f, g\rangle=\sum_{x \in Z}\langle f(x), g(x)\rangle_{W} \quad\left(f, g \in \ell^{2}(Z, W)\right) .
$$

For a non-negative integer $n$, a nonzero vector $u \in \ell^{2}(Z, W)$ and a point $x \in Z$, we define the quantity $p_{n}(u ; x)$ by

$$
p_{n}(u ; x)=\left\|\left(U^{n} u\right)(x)\right\|_{W}^{2},
$$

where $\|\cdot\|_{W}$ denotes the norm on $W$ defined by the fixed Hermitian inner product. It is straightforward to see that the sum of $p_{n}(u ; x)$ over all $x \in Z$ equals $\|u\|^{2}$, the norm square of $u$ in $\ell^{2}(Z, W)$, and hence $p_{n}(u ; x)$ defines a probability distribution on $V$ when $\|u\|=1$. For this reason, we call, in this note, $p_{n}(u ; x)$ the transition probability for the unitary transition operator $U$ with the initial state $u$ even when $u$ is not a unit vector.

Definition 1.1. The transition probability of the unitary transition operator $U$ with initial state $u$ is said to be localized at a point $x \in Z$ if $\limsup _{n \rightarrow \infty} \times$ $p_{n}(u ; x)>0$.

In this paper, we mainly consider the integer lattice $\mathbb{Z}^{d}$ of rank $d \geq 1$ for the set $Z$ and the periodic unitary transition operators on $H=\ell^{2}\left(\mathbb{Z}^{d}, W\right)$ for the unitary operator $U$, which is defined as follows. For each $y \in \mathbb{Z}^{d}$ and $\phi \in W$, we define 
Eigenvalues, absolute continuity and localizations for periodic unitary transition operators

$\delta_{y} \otimes \phi \in H$ by the following formula:

$$
\left(\delta_{y} \otimes \phi\right)(x)= \begin{cases}\phi & (\text { when } x=y), \\ 0 & \text { (otherwise) }\end{cases}
$$

Definition 1.2. A unitary operator $U$ on $H=\ell^{2}\left(\mathbb{Z}^{d}, W\right)$ is said to be a periodic unitary transition operator if the following two conditions are satisfied:

- The unitary operator $U$ commutes with the natural action of the abelian group $\mathbb{Z}^{d}$ on $H$.

- There exists a finite set $S \subset \mathbb{Z}^{d}$, called the set of steps, such that for any $x \in \mathbb{Z}^{d}$, $y \in \mathbb{Z}^{d} \backslash(x+S)$ and any $\phi \in W$, we have $U\left(\delta_{x} \otimes \phi\right)(y)=0$.

It is obvious that unitary transition operators in the usual model of discretetime quantum walks with constant coin matrices and products of finite number of such operators are periodic unitary transition operators. To analyze periodic unitary transition operators, it is natural to use the Fourier transform because of their invariance under the action of $\mathbb{Z}^{d}$. Let $T^{d}$ be the $d$-dimensional torus in $\mathbb{C}^{d}$ defined by

$$
T^{d}=\left\{z=\left(z_{1}, \ldots, z_{d}\right) \in \mathbb{C}^{d} ;\left|z_{j}\right|=1(j=1, \ldots, d)\right\} .
$$

Let $\mathcal{H}=L^{2}\left(T^{d}, W\right)$ be the Hilbert space consisting of all square integrable functions on $T^{d}$ (with respect to the Lebesgue measure on $T^{d}$ ) with values in $W$. The normalized Lebesgue measure on $T^{d}$ is denoted by $\nu_{d}$. The inner product on $\mathcal{H}$ is defined in a usual manner with the Hermitian inner product on $W$. Let $\mathcal{F}: \mathcal{H} \rightarrow H$ be the Fourier transform defined by

$$
\mathcal{F}(f)(x)=\int_{T^{d}} z^{x} f(z) d \nu_{d}(z) \quad(f \in \mathcal{H}),
$$

where we write $z^{x}=z_{1}^{x_{1}} \cdots z_{d}^{x_{d}}$ for a point $z=\left(z_{1}, \ldots, z_{d}\right)$ in the complex torus $T_{\mathbb{C}}^{d}=(\mathbb{C} \backslash\{0\})^{d}$ and a lattice point $x=\left(x_{1}, \ldots, x_{d}\right)$ in $\mathbb{Z}^{d}$. The Fourier transform $\mathcal{F}$ is a unitary operator with the inverse $\mathcal{F}^{*}=\mathcal{F}^{-1}: H \rightarrow \mathcal{H}$ given by

$$
\left(\mathcal{F}^{*} g\right)(z)=\sum_{x \in \mathbb{Z}^{d}} g(x) z^{-x} \quad\left(z \in T^{d}\right) .
$$

For a periodic unitary transition operator $U$, we define a unitary operator $\mathcal{U}$ on $\mathcal{H}$ by $\mathcal{U}=\mathcal{F}^{*} U \mathcal{F}$. Then, the unitary operator $\mathcal{U}$ can be written in the form

$$
(\mathcal{U} f)(z)=\widehat{U}(z) f(z) \quad\left(f \in \mathcal{H}, z \in T^{d}\right)
$$

with a unitary operator $\widehat{U}(z)$ on the finite-dimensional vector space $W$ given by

$$
\widehat{U}(z) \phi=\mathcal{U}(1 \otimes \phi)(z) \quad(\phi \in W),
$$

where for a scalar function $f \in L^{2}\left(T^{d}\right)$ and a vector $\phi \in W$, we define $f \otimes \phi \in \mathcal{H}$ by

$$
(f \otimes \phi)(z)=f(z) \phi \quad\left(z \in T^{d}\right) .
$$

Our main theorem is then described as follows. 
Theorem 1.3. For a periodic unitary transition operator $U$ on $\ell^{2}\left(\mathbb{Z}^{d}, W\right)$, we have the following:

(1) $U$ has an eigenvalue $\omega$ if and only if the matrix-valued function $\widehat{U}(z)$ on $T^{d}$ has an eigenvalue $\omega$ for all points $z$ in $T^{d}$. Hence, the number of eigenvalues of $U$ is finite.

(2) The continuous spectrum of $U$ is absolutely continuous with respect to the Lebesgue measure on the unit circle $S^{1}$ in $\mathbb{C}$.

Therefore, by the absolute continuity of the continuous spectrum, we have the following corollary.

Corollary 1.4. The periodic unitary transition operator $U$ has no eigenvalues if and only if we have $\limsup _{n \rightarrow \infty} p_{n}(u ; x)=0$ for any $u \in \ell^{2}\left(\mathbb{Z}^{d}, W\right)$ and $x \in$ $\mathbb{Z}^{d}$. Therefore, the localization phenomenon does not happen to the quantum walks defined by such unitary operators.

A simple observation shows the following corollary.

Corollary 1.5. Suppose that the periodic unitary transition operator $U$ has only one eigenvalue $\omega$, and let $\pi$ be the orthogonal projection onto the eigenspace corresponding to the eigenvalue $\omega$. Then, for any $u \in H$ and $x \in \mathbb{Z}^{d}$, the limit $\lim _{n \rightarrow \infty} p_{n}(u ; x)$ exists and the following formula holds:

$$
\lim _{n \rightarrow \infty} p_{n}(u ; x)=\|(\pi u)(x)\|_{W}^{2} .
$$

It would be necessary here to give some remarks on these results. First, it was pointed out to the author by professor Alain Joye that the singular continuous spectrum of (general unitary operator) $U$ does not affect the long-time average of the transition probabilities,

$$
\bar{p}(u ; x)=\lim _{N \rightarrow \infty} \frac{1}{N} \sum_{n=1}^{N} p_{n}(u ; x) .
$$

Indeed, let $U$ be a general unitary operator on $\ell^{2}(Z, W)$ (for a general countable set $Z$ ) and let $\pi_{\lambda}$ be the orthogonal projection onto the eigenspace corresponding to an eigenvalue $\lambda$ of $U$. Then, Wiener's formula ${ }^{16}$ tells us that the limit $\bar{p}(u ; x)$ exists and satisfies

$$
\bar{p}(u ; x)=\sum_{\lambda: \text { eigenvalue of } U}\left\|\left(\pi_{\lambda} u\right)(x)\right\|_{W}^{2} .
$$

From this it is obvious that if $U$ has an eigenvalue then a localization takes place at some point $x \in Z$ and an initial state $u \in \ell^{2}(Z, W)$. In Corollary 1.5 we take the long-time limit (but not the average) itself of the transition probability under the assumption that $U$ has only one eigenvalue. For example, it is well known that the three-state Grover walk on $\mathbb{Z}$ and the square of the two-dimensional Grover walk satisfy this assumption. 
The absolute continuity of continuous spectrum for certain class of self-adjoint operators has been obtained in the work of Gérard-Nier ${ }^{7}$ The theory developed in Ref. 7 is very powerful, and it might be possible to apply this theory to the generator of the periodic unitary transition operators. However, taking a logarithm of the periodic unitary transition operators would cause singularities of the function corresponding to $\widehat{U}$ introduced above and hence it does not seem so straightforward to apply it. Since our situation is rather easier, it would be reasonable to give a direct and constructive proof of absolute continuity.

Since in the setting up described above the vector space $W$ can be arbitrarily chosen, Theorem 1.3 is applicable to certain unitary operators on topological crystal over finite graphs. As is discussed in Sec. 2, some unitary transition operators are defined over the set of oriented edges. But, the set of vertices, as in the classical case, might be used to formulate quantum system over a topological crystal. Therefore, we use a setting described as follows. As before, let $Z$ be a countable set and let $W_{0}$ be a finite-dimensional Hilbert space with the inner product $\langle\cdot, \cdot\rangle_{W_{0}}$. Suppose that a free abelian group $\Gamma$ of finite rank $d$ acts on $Z$ freely and the quotient set $Z_{0}=Z / \Gamma$ is finite. Let $p: Z \rightarrow Z_{0}$ be the natural projection. Identifying the finite set $Z_{0}$ with a fixed fundamental set $F_{0}$ of the action of $\Gamma$, we may regard $Z_{0}$ as a subset of $Z$. Let $C\left(Z, W_{0}\right)$ be the vector space of all function from $Z$ to $W_{0}$. Let $U$ be a unitary operator on the Hilbert space $\ell^{2}\left(Z, W_{0}\right)$ satisfying the following two conditions:

(i) $U$ commutes with the action of $\Gamma$ on $\ell^{2}\left(Z, W_{0}\right)$.

(ii) There exists a finite set $S \subset \Gamma$ such that, for any $x_{0}, y_{0} \in Z_{0}, \alpha \in \Gamma, \beta \in \Gamma \backslash \alpha S$ and $\phi \in W_{0}$, we have $U\left(\delta_{\alpha x_{0}} \otimes \phi\right)\left(\beta y_{0}\right)=0$.

Here, we note that the group operation on $\Gamma$ is written as a multiplication because it would be better to describe the action of $\Gamma$ on $Z$. Condition (ii) means that if the quantum walker with the time evolution $U$ start at a point in the fundamental set, $\alpha F_{0}$, the walker can arrive in one step at points in $\bigcup_{\gamma \in S} \gamma \alpha F_{0}$. Thus, the finite set $S$ depends on the choice of the fundamental set $F_{0}$. Let $\widehat{\Gamma}$ be the group of $U(1)$-character on $\Gamma$. For each $\chi \in \widehat{\Gamma}$, we set

$$
C_{\chi}\left(Z, W_{0}\right)=\left\{f: Z \rightarrow W_{0} ; f(\alpha x)=\chi(\alpha) f(x)(x \in Z, \alpha \in \Gamma)\right\} .
$$

The space $C_{\chi}\left(Z, W_{0}\right)$ is finite-dimensional and is isomorphic to $\ell^{2}\left(Z_{0}, W_{0}\right)$, and it is equipped with a natural Hermitian inner product inherited from $\ell^{2}\left(Z_{0}, W_{0}\right)$. By the assumption (i), (ii), the operator $U$ is continuous with respect to the topology of point-wise convergence, and thus it defines a linear map on the space of all $W_{0^{-}}$ valued function on $Z$. For each $\chi \in \widehat{\Gamma}$, we define a unitary operator $U_{\chi}$ on $C_{\chi}\left(Z, W_{0}\right)$ as the restriction of $U$ to $C_{\chi}\left(Z, W_{0}\right)$.

Corollary 1.6. Let $U$ be a unitary operator on $\ell^{2}\left(Z, W_{0}\right)$ satisfying the above two conditions and, for $\chi \in \widehat{\Gamma}$, let $U_{\chi}$ be the unitary operator on $C_{\chi}\left(Z, W_{0}\right)$ defined as 
above. Then, we have the following:

(1) $U$ has an eigenvalue $\omega$ if and only if the unitary operator $U_{\chi}$ on $C_{\chi}\left(Z, W_{0}\right)$ has an eigenvalue $\omega$ for all points $\chi \in \widehat{\Gamma}$. Hence, the number of eigenvalues of $U$ is finite.

(2) The continuous spectrum of $U$ is absolutely continuous with respect to the Lebesgue measure on the unit circle $S^{1}$ in $\mathbb{C}$.

Hence, the statements in Corollaries 1.4 and 1.5 holds true for $U$.

Organization of this paper is as follows. First, in Sec. 2, the corollaries are proved. Main reason for applicability of Theorem 1.3 to the setting-up in Corollary 1.6 is that, under the assumption (i), (ii), the operator $U$ becomes a periodic unitary transition operator on $\ell^{2}\left(Z, W_{0}\right) \cong \ell^{2}(\Gamma, W)$ with $W=\ell^{2}\left(Z_{0}, W_{0}\right)$. An example, the Grover walks on topological crystals will be explained in Sec. 2 In Sec. 3 some simple but important properties of periodic unitary transition operators are summarized. The item (1) in Theorem 1.3 is an easy consequence of the formula (1.5), but its proof is written, for completeness of the presentation, in Sec. 4 In Sec. 5, absolute continuity of the continuous spectrum is discussed. In this section, we employ the analytic perturbation theory developed by Baumgärte 4 and use a coarea formula to construct a density function of the spectral measures.

\section{Proofs of Corollaries}

Let $Z$ be, as in Sec. 1 a countable set and let $W$ be a complex vector space of dimension $D$ with a fixed Hermitian inner product $\langle\cdot, \cdot\rangle_{W}$. Let $U$ be a unitary operator on the Hilbert space $H=\ell^{2}(Z, W)$ with the inner product given by (1.1). Let $\operatorname{spec}(U)$ and $\operatorname{spec}(U)_{p}$ denote the spectrum and the set of eigenvalues of $U$, respectively. Since $U$ is unitary, $\operatorname{spec}(U)$ is contained in the unit circle $S^{1}$ in the complex plane, and the spectral resolution of $U$ takes the form

$$
U=\int_{S^{1}} \lambda d E(\lambda)
$$

where $E$ is a projection-valued measure on $S^{1}$. If $\omega \in \operatorname{spec}(U)_{p}$, then $\pi_{\omega}=E(\{\omega\})$ is the projection onto the eigenspace corresponding to $\omega$. We say that $u \in H$ has absolutely continuous spectral measure if the measure $\|E(\cdot) u\|^{2}$ is absolutely continuous with respect to the Lebesgue measure on the circle. The following lemma can be proved by using the Riemann-Lebesgue lemma.

Lemma 2.1. Let $u \in H$. Suppose that $u$ has absolutely continuous spectral measure. Then, for any $x \in Z$, we have $\lim _{n \rightarrow \infty} p_{n}(u ; x)=0$.

Using Lemma 2.1, it is easy to show the following lemma.

Lemma 2.2. Suppose that a unitary operator $U$ on $H$ has only one eigenvalue and that the continuous spectrum of $U$ is absolutely continuous. Let $\pi$ be the orthogonal 
projection onto the eigenspace of $U$. Then, we have $\lim _{n \rightarrow \infty} p_{n}(u ; x)=\|(\pi u)(x)\|_{W}^{2}$ for any $u \in H$ and $x \in Z$.

Proof. The transition probability $p_{n}(u ; x)$ can be written as

$$
\begin{aligned}
p_{n}(u ; x) & =p_{n}(\pi u ; x)+p_{n}\left(\pi_{c} u ; x\right)+r_{n}(u ; x) \\
& =\|(\pi u)(x)\|_{W}^{2}+p_{n}\left(\pi_{c} u ; x\right)+r_{n}(u ; x),
\end{aligned}
$$

where we set $\pi_{c}=I-\pi$ and the quantity $r_{n}(u ; x)$ is defined by

$$
r_{n}(u ; x)=2 \operatorname{Re}\left(\left\langle\left(U^{n} \pi u\right)(x),\left(U^{n} \pi_{c} u\right)(x)\right\rangle_{W}\right) .
$$

By the assumption on the absolute continuity and Lemma 2.1, we see $\lim _{n \rightarrow \infty} \times$ $p_{n}\left(\pi_{c} u ; x\right)=0$. Cauchy-Schwarz inequality shows $r_{n}(w ; x) \leq 2\|\pi w(x)\|_{\mathbb{C}^{D}} \times$ $p_{n}\left(\pi_{c} w ; x\right)^{1 / 2}$ which tends to zero as $n \rightarrow \infty$.

Corollaries 1.4 and 1.5 are direct consequences of the Wiener formula (1.9), Lemmas 2.1 and 2.2 and Theorem 1.3

Next, we give a proof of Corollary 1.6. To begin with, we prepare notation. Let $Z$ be, as before, a countable set and let $\Gamma$ be a free abelian group of finite rank $d$. We suppose that $\Gamma$ acts on $Z$ freely with the finite quotient $Z_{0}=Z / \Gamma$. Let $F_{0} \subset Z$ be a complete set of representatives of elements in the orbit space $Z_{0}$, which is often called a fundamental set of the action of $\Gamma$ on $Z$. The natural projection $p: Z \rightarrow Z_{0}$ maps $F_{0}$ bijectively onto $Z_{0}$. We set $q=\left(\left.p\right|_{F_{0}}\right)^{-1}: Z_{0} \rightarrow F_{0}$. Let $U$ be a unitary operator on $\ell^{2}\left(Z, W_{0}\right)$ satisfying conditions (i), (ii) described before Corollary 1.6. Define the operator $r: \ell^{2}\left(Z, W_{0}\right) \rightarrow \ell^{2}\left(\Gamma, \ell^{2}\left(Z_{0}, W_{0}\right)\right)$ by

$$
r(f)(\alpha)\left(x_{0}\right)=f\left(\alpha q\left(x_{0}\right)\right) \quad\left(f \in \ell^{2}\left(Z, W_{0}\right), \alpha \in \Gamma, x_{0} \in Z_{0}\right) .
$$

The operator $r$ is unitary with the natural Hermitian inner product on $\ell^{2}\left(\Gamma, \ell^{2}\left(Z_{0}, W_{0}\right)\right)$. Since the action of $\Gamma$ on $\ell^{2}\left(Z, W_{0}\right)$ is intertwined by the unitary operator $r: \ell^{2}\left(Z, W_{0}\right) \rightarrow \ell^{2}\left(\Gamma, \ell^{2}\left(Z_{0}, W_{0}\right)\right)$ with the natural action of $\Gamma$ on $\ell^{2}\left(\Gamma, \ell^{2}\left(Z_{0}, W_{0}\right)\right)$, the unitary operator $r U r^{*}$ on $\ell^{2}\left(\Gamma, \ell^{2}\left(Z_{0}, W_{0}\right)\right)$ is still $\Gamma$-equivariant.

Lemma 2.3. The unitary operator $r U r^{*}$ on $\ell^{2}\left(\Gamma, \ell^{2}\left(Z_{0}, W_{0}\right)\right)$ is a periodic unitary transition operator in the sense of Definition [1.2.

Proof. Condition (i) for $U$ ensures that $r U r^{*}$ commutes with the action of $\Gamma$ on $\ell^{2}\left(\Gamma, \ell^{2}\left(Z_{0}, W_{0}\right)\right)$. We take a finite set $S \subset \Gamma$ as in condition (ii) imposed on $U$. Let $\alpha \in \Gamma$ and $\beta \in \Gamma \backslash \alpha S$, and let $f_{0} \in \ell^{2}\left(Z_{0}, W_{0}\right)$ be arbitrary. We have to compute $r U r^{*}\left(\delta_{\alpha} \otimes f_{0}\right)(\beta)$. The function $\delta_{\alpha} \otimes f_{0}$ is in $\ell^{2}\left(\Gamma, \ell^{2}\left(Z_{0}, W_{0}\right)\right)$, and the function in $\ell^{2}\left(Z, W_{0}\right)$ corresponding to it is given by

$$
r^{*}\left(\delta_{\alpha} \otimes f_{0}\right)=\sum_{x_{0} \in Z_{0}} \delta_{\alpha q\left(x_{0}\right)} \otimes f_{0}\left(x_{0}\right) .
$$


Thus, we get

$$
U r^{*}\left(\delta_{\alpha} \otimes f_{0}\right)=\sum_{x_{0} \in Z_{0}} U\left(\delta_{\alpha q\left(x_{0}\right)} \otimes f_{0}\left(x_{0}\right)\right) .
$$

Condition (ii) imposed on $U$ implies that, for $\beta \notin \alpha S$ and $y_{0} \in Z_{0}$, the value at $\beta q\left(y_{0}\right)$ of each of the summand in the right-hand side vanishes. Hence $r U r^{*}\left(\delta_{\alpha} \otimes\right.$ $\left.f_{0}\right)(\beta)=0$ for each $\beta \notin \alpha S$, which shows that $r U r^{*}$ is a periodic unitary transition operator on $\ell^{2}\left(\Gamma, \ell^{2}\left(Z_{0}, W_{0}\right)\right)$.

The map $r$ is naturally extended to the map $r: C\left(Z, W_{0}\right) \rightarrow C\left(\Gamma, \ell^{2}\left(Z_{0}, W_{0}\right)\right)$. Since $r$ commutes with the action of $\Gamma$, we have the following isomorphism:

$$
r: C_{\chi}\left(Z, W_{0}\right) \stackrel{\cong}{\rightarrow} \chi \otimes \ell^{2}\left(Z_{0}, W_{0}\right)
$$

where for a function $\mu$ on $\Gamma$ and $f_{0} \in \ell^{2}\left(Z_{0}, W_{0}\right)$, the function $\mu \otimes f_{0} \in$ $C\left(\Gamma, \ell^{2}\left(Z_{0}, W_{0}\right)\right)$ is defined by

$$
\left(\mu \otimes f_{0}\right)(\alpha)\left(x_{0}\right)=\mu(\alpha) f_{0}\left(x_{0}\right) \quad\left(\alpha \in \Gamma, x_{0} \in Z_{0}\right) .
$$

The map $r$ on $C_{\chi}\left(Z, W_{0}\right)$ composed with the map

$$
\chi \otimes \ell^{2}\left(Z_{0}, W_{0}\right) \ni \chi \otimes f_{0} \mapsto f_{0}=\left(\chi \otimes f_{0}\right)(1) \in \ell^{2}\left(Z_{0}, W_{0}\right)
$$

gives a unitary operator

$$
r_{\chi}: C_{\chi}\left(Z, W_{0}\right) \stackrel{\cong}{\rightarrow} \ell^{2}\left(Z_{0}, W_{0}\right), \quad r_{\chi}(f)=q^{*} f .
$$

The Fourier transform $\mathcal{F}: L^{2}\left(\widehat{\Gamma}, \ell^{2}\left(Z_{0}, W_{0}\right)\right) \rightarrow \ell^{2}\left(\Gamma, \ell^{2}\left(Z_{0}, W_{0}\right)\right)$ and its inverse is given by

$$
(\mathcal{F} f)(\alpha)\left(x_{0}\right)=\int_{\widehat{\Gamma}} \rho(\alpha) f(\rho)\left(x_{0}\right) d \nu(\rho), \quad\left(\mathcal{F}^{-1} g\right)(\chi)\left(x_{0}\right)=\sum_{\gamma \in \Gamma} \chi\left(\gamma^{-1}\right) g(\gamma)\left(x_{0}\right)
$$

where $f \in L^{2}\left(\widehat{\Gamma}, \ell^{2}\left(Z_{0}, W_{0}\right)\right), g \in \ell^{2}\left(\Gamma, \ell^{2}\left(Z_{0}, W_{0}\right)\right), \chi \in \widehat{\Gamma}, \alpha \in \Gamma$ and $x_{0} \in Z_{0}$.

Lemma 2.4. For each $\chi \in \widehat{\Gamma}$, we have $\widehat{r U r^{*}}(\chi)=r_{\chi} U_{\chi} r_{\chi}^{*}$, where $U_{\chi}$ is the restriction of $U$ on $C_{\chi}\left(Z, W_{0}\right)$.

Proof. We take $f_{0} \in \ell^{2}\left(Z_{0}, W_{0}\right)$. Then, we have $\mathcal{F}\left(\mathbf{1} \otimes f_{0}\right)=\delta_{1} \otimes f_{0}$, where 1 is the function taking the value identically 1 on $\widehat{\Gamma}$ and $\delta_{1}$ is the delta function at the identity element $1 \in \Gamma$. We see, for $\chi \in \widehat{\Gamma}$,

$$
\begin{aligned}
\widehat{r U r^{*}}(\chi) f_{0} & =\mathcal{U}\left(1 \otimes f_{0}\right)(\chi)=\sum_{\gamma \in \Gamma} \chi(\gamma) r U r^{*}\left(1 \otimes f_{0}\right)\left(\gamma^{-1}\right) \\
& =\sum_{\gamma \in \Gamma} \chi(\gamma) r U r^{*}\left(\delta_{\gamma} \otimes f_{0}\right)(1) .
\end{aligned}
$$

Now, the sum $\sum_{\gamma \in \Gamma} \chi(\gamma) \delta_{\gamma} \otimes f_{0}$ converges in the topology of point-wise convergence on $C\left(\Gamma, \ell^{2}\left(Z_{0}, W_{0}\right)\right)$ to the function $\chi \otimes f_{0}$. Since, by condition (ii), $r U r^{*}$ is 
continuous in this topology, we have

$$
\sum_{\gamma \in \Gamma} \chi(\gamma) r U r^{*}\left(\delta_{\gamma} \otimes f_{0}\right)=r U r^{*}\left(\chi \otimes f_{0}\right)=r_{\chi} U_{\chi} r_{\chi}^{*} f_{0},
$$

which completes the proof.

Now, Corollary 1.6 follows from Lemmas 2.3 2.4 and Theorem 1.3

We remark that, in the above proof, the space $Z$ is regarded as a product $\Gamma \times Z_{0}$. This method is, for the setting-up in graph theory, not quite natural because it does not take the graph structure into account. For the setting in graph theory, it would be more transparent to use a unitary operator constructed as follows. We fix a function $s_{\chi}: Z \rightarrow U(1)$ satisfying $s_{\chi}(\alpha x)=\chi(\alpha) s_{\chi}(x)$ for all $\alpha \in \Gamma, x \in Z$. Then the operator $S_{\chi}: \ell^{2}\left(Z_{0}, W_{0}\right) \rightarrow C_{\chi}\left(Z, W_{0}\right)$ given by

$$
\left(S_{\chi} f_{0}\right)(x)=s_{\chi}(x) f(p(x)) \quad\left(f_{0} \in \ell^{2}\left(Z_{0}, W_{0}\right), x \in Z\right)
$$

is a unitary operator. In our setting above, we formulated problems on $\ell^{2}(\Gamma$, $\left.\ell^{2}\left(Z_{0}, W_{0}\right)\right)$ rather than on $\ell^{2}\left(Z, W_{0}\right)$. Thus it would be reasonable and useful to use the operator $r_{\chi}: C_{\chi}\left(Z, W_{0}\right) \rightarrow \ell^{2}\left(Z_{0}, W_{0}\right)$. The operator $S_{\chi}^{-1} U_{\chi} S_{\chi}$ is unitarily equivalent to $r_{\chi} U_{\chi} r_{\chi}^{*}$ by the operator $r_{\chi} S_{\chi}$ which is just the multiplication by the function $q^{*} s_{\chi}$. Therefore, the spectral structure of $U_{\chi}$ or $\widehat{r U r^{*}}(\chi)$ is the same as that of $S_{\chi}^{-1} U_{\chi} S_{\chi}$.

Let us describe an example where Corollary 1.6 can be applied, Grover walks on topological crystals. In the following, the terminology, definitions and the descriptions basically follow that of Refs. 19 and 22 For a graph $Y$, we denote the set of vertices and the set of oriented edges by $V(Y), E(Y)$, respectively. For any $e \in E(Y)$, the origin $o(e)$ and the terminus $t(e)$ are the vertices which are connected by the oriented edge $e$ in an obvious direction. For any vertex $y \in V(Y)$, we set $E(Y)_{y}=\{e \in E(Y) ; o(e)=x\}$. Then the degree of $y \in V(Y)$, denoted by $\operatorname{deg}(y)$, is given by the number of elements in $E(Y)_{y}$. An orientation of the graph $Y=(V(Y), E(Y))$ is a subset $E^{o}(Y)$ of $E(Y)$ such that $E(Y)$ is written as the disjoint union $E(Y)=E^{o}(Y) \cup \overline{E^{o}(Y)}$.

Let $X$ be a topological crystal ${ }^{22}$ over a connected finite graph $X_{0}$ with an abstract periodic lattice $\Gamma$, namely, $X$ is an infinite locally finite connected graph on which a free abelian group $\Gamma$ of rank $d(0<d<+\infty)$ acts freely and without inversion such that the quotient graph is $X_{0}$. The natural projection from $X$ onto $X_{0}$ is denoted by $p: X \rightarrow X_{0}$. A linear map $U$ on the space $C(E(X))$ consisting of all complex-valued function on $E(X)$ defined by

$$
(U \phi)(e)=-\phi(\bar{e})+\frac{2}{\operatorname{deg}(t(e))} \sum_{e^{\prime} \in E(X)_{t(e)}} \phi\left(e^{\prime}\right) \quad(\phi \in C(E(X)), e \in E(X)),
$$

is often called a Grover walk ${ }^{25}$ See also Ref. 9, where a generalization, called a twisted Szegedy walk is formulated. The restriction of $U$ on $\ell^{2}(E(X))$, which is also denoted by $U$, defines a unitary operator on $\ell^{2}(E(X))$, and $U$ commutes with the 
action of $\Gamma$. Since the value $(U \phi)(e)$ depends only on $\phi\left(e^{\prime}\right)$ and $\phi(\bar{e})$ with the edges $e^{\prime}$ adjacent to $e$, the operator $U$ satisfies conditions (i), (ii) described before the statement of Corollary 1.6 .

Let us fix $\chi \in \widehat{\Gamma}$ and compute the operator $\widehat{U}(\chi) \cong U_{\chi}$ on $\ell^{2}\left(E\left(X_{0}\right)\right)$. To compute it, we need to choose a fundamental set $F_{0}$ in the set of oriented edges $E(X)$ of the action of $\Gamma$. We fix a spanning tree $T_{0}$ in $X_{0}$ and its lift $T$ in $X$. For any $u \in E\left(X_{0}\right)$, there exists a unique edge $q(u) \in E(X)$ with the properties $p(q(u))=u$ and $o(q(u)) \in V(T)$. Then, the finite set $F_{0}=q\left(E\left(X_{0}\right)\right)$ in $E(X)$ is a fundamental set of the action of $\Gamma$ on $E(X)$. Note that in general $q(\bar{u}) \neq \overline{q(u)}$. But if $u \in E\left(T_{0}\right)$ then we have $q(\bar{u})=\overline{q(u)}$. We follow the construction of a unitary operator $S_{\chi}: \ell^{2}\left(E\left(X_{0}\right)\right) \rightarrow C_{\chi}(X)$ given in Ref. 19. We fix $\omega \in \operatorname{Hom}(\Gamma, \mathbb{R})$ such that $\chi=\exp (2 \pi \sqrt{-1} \omega)$. Let $\mu: H_{1}\left(X_{0}, \mathbb{Z}\right) \rightarrow \Gamma$ be the surjective homomorphism characterized by the identity

$$
t(\tilde{c})=\mu(\langle c\rangle) o(\tilde{c})
$$

where $c$ is a closed path in $X_{0}, \tilde{c}$ is a lift of $c,\langle c\rangle$ is the homology class of $c$ (with coefficients in $\mathbb{Z}$ ) and $t(\tilde{c}), o(\tilde{c})$ denote the terminus and origin of the path $\tilde{c}$ in $X$. We extend $\mu$ as a linear map $\mu_{\mathbb{R}}: H_{1}\left(X_{0}, \mathbb{R}\right) \rightarrow \Gamma \otimes \mathbb{R}$. Since the dual space of $H_{1}\left(X_{0}, \mathbb{R}\right)$ is naturally identified with the cohomology group $H^{1}\left(X_{0}, \mathbb{R}\right)$, the transpose $\mu_{\mathbb{R}}^{*}: \operatorname{Hom}(\Gamma, \mathbb{R}) \cong(\Gamma \otimes \mathbb{R})^{*} \rightarrow H^{1}\left(X_{0}, \mathbb{R}\right)$ is an injective linear map. We take a one-form $\eta$, which is a function on $E\left(X_{0}\right)$ such that $\eta(\bar{e})=-\eta(e)$, whose cohomology class is $\mu_{\mathbb{R}}^{*} \omega \in H^{1}\left(X_{0}, \mathbb{R}\right)$. We have $\omega(\mu(\langle c\rangle))=\eta(c)$ for any closed path $c$, where for $c=\left(e_{1}, \ldots, e_{n}\right)$, we write $\eta(c)=\sum_{i=1}^{n} \eta\left(e_{i}\right)$. We remark that $\eta(c)$ does not depend on the choice of $\eta$ representing the cohomology class $\mu_{\mathbb{R}}^{*} \omega$. We fix $e_{*} \in E(T)$ and define a function $s_{\eta}: E(X) \rightarrow \mathbb{C}$ by

$$
s_{\eta}(e)=e^{2 \pi \sqrt{-1}\left(p^{*} \eta\right)\left(\gamma\left(e_{*}, e\right)\right)},
$$

where $\gamma\left(e_{*}, e\right)$ denotes any path from $o\left(e_{*}\right)$ to $t(e)$ in $X$ such that the first edge of $\gamma\left(e_{*}, e\right)$ is $e_{*}$ and the last edge is $e$. Since $\mu(\langle p(\tilde{c})\rangle)=1$ for any closed path $\tilde{c}$ on $X$, the right-hand side in the definition of $s_{\eta}$ does not depend on the choice of such a path from $o\left(e_{*}\right)$ to $t(e)$, and we have $s_{\eta} \in C_{\chi}(E(X))$. We note that $s_{\eta}$ depends on the choice of 1 -form $\eta$ representing $\mu_{\mathbb{R}}^{*} \omega$ and even the choice of $\omega$. Now, the linear $\operatorname{map} S_{\chi}: \ell^{2}\left(E\left(X_{0}\right)\right) \rightarrow C_{\chi}(E(X))$ defined by

$$
\left(S_{\chi} f_{0}\right)(e)=s_{\eta}(e) f_{0}(p(e)) \quad\left(f_{0} \in \ell^{2}\left(E\left(X_{0}\right)\right), e \in E(X)\right)
$$

is a unitary isomorphism. It is straightforward to see

$$
\begin{aligned}
\left(S_{\chi}^{-1} U_{\chi} S_{\chi} f_{0}\right)\left(e_{0}\right)= & -\left(e^{2 \pi \sqrt{-1} \eta} f_{0}\right)\left(\overline{e_{0}}\right) \\
& +\frac{2}{\operatorname{deg} t\left(e_{0}\right)} \sum_{u \in E\left(X_{0}\right)_{t\left(e_{0}\right)}}\left(e^{2 \pi \sqrt{-1} \eta} f_{0}\right)(u) \quad\left(e_{0} \in E\left(X_{0}\right)\right),
\end{aligned}
$$

which is (unitarily equivalent to) the twisted Szegedy walk whose spectral structure is discussed in Ref. 9 


\section{Simple Properties of Periodic Unitary Transition Operators}

For $i=1, \ldots, d$, let $\tau_{i}$ be the shift operator on $H=\ell^{2}\left(\mathbb{Z}^{d}, \mathbb{C}^{D}\right)$ defined by

$$
\left(\tau_{i} g\right)(x)=g\left(x-e_{i}\right) \quad\left(g \in H, x \in \mathbb{Z}^{d}\right),
$$

where $\left\{e_{1}, \ldots, e_{d}\right\}$ denotes the standard basis of $\mathbb{Z}^{d}$ over $\mathbb{Z}$. Then, $\tau_{i}$ 's are unitary operators on $H$. We note that $\tau_{i}$ and $\tau_{j}$ commutes for any $i, j$, and hence, for $\alpha=\sum_{i=1}^{d} \alpha_{i} e_{i}$, we may write $\tau^{\alpha}=\tau_{1}^{\alpha_{1}} \cdots \tau_{d}^{\alpha_{d}}$. The action of $\mathbb{Z}^{d}$ on $H$ is given by the operators $\tau^{\alpha}$.

Lemma 3.1. Let $U$ be a periodic unitary transition operator on $H$ with the set of steps $S \subset \mathbb{Z}^{d}$. For each $\alpha \in S$, define a linear map $C(\alpha)$ on $\mathbb{C}^{D}$ by

$$
C(\alpha) \phi=U\left(\delta_{0} \otimes \phi\right)(\alpha) \quad\left(\phi \in \mathbb{C}^{D}\right) .
$$

Then, $U$ can be written in the following form:

$$
U=\sum_{\alpha \in S} \tau^{\alpha} C(\alpha)
$$

Proof. It is straightforward to see that, for any $\phi \in \mathbb{C}^{D}$ and $x \in \mathbb{Z}^{d}$,

$$
U\left(\delta_{x} \otimes \phi\right)=\sum_{\alpha \in S} \tau^{\alpha} \delta_{x} \otimes C(\alpha) \phi=\left(\sum_{\alpha \in S} \tau^{\alpha} C(\alpha)\right)\left(\delta_{x} \otimes \phi\right) .
$$

Since the set $\left\{\delta_{x} \otimes \phi\right\}_{x \in \mathbb{Z}^{d}, \phi \in \mathbb{C}^{D}}$ spans $H$, we conclude the assertion.

The operator $\mathcal{F}^{*} \tau_{i} \mathcal{F}$, where $\mathcal{F}: \mathcal{H} \rightarrow H$ is the Fourier transform defined in (1.3) and $\mathcal{F}^{*}$ is its inverse, is the multiplication operator defined by the coordinate function $z_{i}$, where $z=\left(z_{1}, \ldots, z_{d}\right) \in T^{d}$. Hence, the operator $\mathcal{U}=\mathcal{F}^{*} U \mathcal{F}$ is written in the form 1.5 with the matrix $\widehat{U}(z)$ given by the formula

$$
\widehat{U}(z) \phi=\sum_{\alpha \in S} z^{\alpha} C(\alpha) \phi=\mathcal{U}(1 \otimes \phi)(z) \quad\left(\phi \in \mathbb{C}^{D}\right) .
$$

Therefore, by Lemma 3.1 and the assumption that $U$ is a unitary operator, we see

$$
\sum_{\alpha, \beta \in S, \alpha-\beta=\gamma} C(\beta)^{*} C(\alpha)=\delta_{\gamma, 0} I
$$

for any $\gamma \in \mathbb{Z}^{d}$. From this and (3.1), it follows that the matrix $\widehat{U}(z)$ is unitary for any $z \in T^{d}$. We note that $\widehat{U}(z)$ is a Laurent polynomial in $z \in T^{d}$ and hence it is naturally extended to the complex torus $T_{\mathbb{C}}^{d}=(\mathbb{C} \backslash\{0\})^{d}$. However, in general, $\widehat{U}(z)$ is not normal when $z$ is not in the real torus $T^{d}$. We remark that, for a bounded operator $A$ on $H=\ell^{2}\left(\mathbb{Z}^{d}, \mathbb{C}^{D}\right)$ commuting with the action of $\mathbb{Z}^{d}$, the bounded operator $\mathcal{F}^{*} A \mathcal{F}$ on $\mathcal{H}=L^{2}\left(T^{d}, \mathbb{C}^{D}\right)$ commutes with multiplication operators by continuous functions on $T^{d}$. The characteristic polynomial

$$
\chi(\zeta, z)=\operatorname{det}(\zeta-\widehat{U}(z)) \quad\left(z \in T_{\mathbb{C}}^{d}, \zeta \in \mathbb{C}\right)
$$

of $\widehat{U}(z)$ is a polynomial in $\zeta$ of degree $D$ with coefficients in the ring of Laurent polynomials in $z \in T_{\mathbb{C}}^{d}$. 
Lemma 3.2. We have the following:

$$
\begin{aligned}
\operatorname{spec}(U)= & \left\{\lambda \in S^{1} ; \text { There exists a point } z \in T^{d} \text { such that } \lambda\right. \\
& \text { is an eigenvalue of } \widehat{U}(z)\} .
\end{aligned}
$$

Proof. It is easy to show that the spectrum $\operatorname{spec}(U)$ is contained in the set in the right-hand side above. Thus, we prove that $\operatorname{spec}(U)$ contains the set in the right-hand side. Suppose that $\lambda$ is in the resolvent set of $U$ and set $V=\{z \in$ $\left.T^{d} ; \chi(\lambda, z) \neq 0\right\}$. Then, $\mathcal{R}=(\lambda-\mathcal{U})^{-1}$ is a bounded operator on $\mathcal{H}$. Let $W \subset T^{d}$ be a Borel set such that $\nu_{d}(W)=1$ and $\widehat{R}(z) \phi:=(\lambda-\mathcal{U})^{-1}(1 \otimes \phi)(z)$ is defined for any $z \in W$ and any $\phi \in \mathbb{C}^{D}$. Then, we have $\phi=(\lambda-\widehat{U}(z)) \widehat{R}(z) \phi$ for any $z \in W$ and $\phi \in \mathbb{C}^{D}$. Thus, $W \subset V$. This shows that $\nu_{d}(V)=1$ and that $V$ is an open dense set such that $T^{d} \backslash V$ does not contain any non-empty open set. Therefore we write $\widehat{R}(z)=(\lambda-\widehat{U}(z))^{-1}$ for $z \in V$. We claim that $V=T^{d}$. To show this, suppose contrary that $T^{d} \backslash V \neq \emptyset$ and we take $z_{o} \in T^{d} \backslash V$. We take $\phi_{o} \in \mathbb{C}^{D}$ such that $\left\|\phi_{o}\right\|_{\mathbb{C}^{D}}=1$ and $\left(\lambda-\widehat{U}\left(z_{o}\right)\right) \phi_{o}=0$. For any positive integer $n$, there exists a neighborhood $U_{n}$ of $z_{o}$ in $T^{d}$ such that $\left\|(\lambda-\widehat{U}(z)) \phi_{o}\right\|_{\mathbb{C}^{D}}<1 /(n+1)$ for any $z \in U_{n}$. Then, for any $z \in U_{n} \cap V$, we have

$$
1=\left\|\widehat{R}(z)(\lambda-\widehat{U}(z)) \phi_{o}\right\|_{\mathbb{C}^{D}} \leq\|\widehat{R}(z)\|_{\mathrm{op}}\left\|(\lambda-\widehat{U}(z)) \phi_{o}\right\|_{\mathbb{C}^{D}} \leq\|\widehat{R}(z)\|_{\mathrm{op}} /(n+1),
$$

where $\|\cdot\|_{\text {op }}$ denotes the operator norm. For each $n$, we fix $z_{n} \in U_{n} \cap V$. One can find $\phi_{n} \in \mathbb{C}^{D}$ with $\left\|\phi_{n}\right\|_{\mathbb{C}^{D}}=1$ such that $n<\left\|\widehat{R}\left(z_{n}\right) \phi_{n}\right\|_{\mathbb{C}^{D}}$. There exists a neighborhood $V_{n}$ of $z_{n}$ such that $V_{n} \subset U_{n} \cap V$ and $n<\left\|\widehat{R}(z) \phi_{n}\right\|_{\mathbb{C}^{D}}$ for any $z \in V_{n}$. We take a continuous function $f_{n}$ on $T^{d}$ such that the support of $f_{n}$ is contained in $V_{n}$ and $\left\|f_{n}\right\|_{L^{2}\left(T^{d}\right)}=1$. We set $v_{n}=f_{n} \otimes \phi_{n}=f_{n}\left(1 \otimes \phi_{n}\right) \in \mathcal{H}$ which satisfies $\left\|v_{n}\right\|=1$. We see $\left(\mathcal{R} v_{n}\right)(z)=f_{n}(z) \widehat{R}(z) \phi_{n}$ for any $z \in W$. Then,

$$
\|\mathcal{R}\|_{\text {op }}^{2} \geq\left\|\mathcal{R} v_{n}\right\|^{2}=\int_{V_{n}}\left|f_{n}(z)\right|^{2}\left\|\widehat{R}(z) \phi_{n}\right\|_{\mathbb{C}^{D}}^{2} d \nu_{d}(z) \geq n^{2},
$$

which contradicts the fact that $\mathcal{R}$ is a bounded operator. This shows that $V=T^{d}$, and hence $\lambda$ is not an eigenvalue of $\widehat{U}(z)$ for all $z \in T^{d}$.

\section{Eigenvalues of Periodic Unitary Transition Operators}

In this section and the next section, we frequently use the following lemma. This lemma is essentially the same as Lemma 4.4 in Ref. 10. However, we give its proof for convenience.

Lemma 4.1. Let $\Delta \subset \mathbb{C}^{d}$ be a domain. Let $f$ be a holomorphic function on $\Delta$. Suppose that $f^{-1}(0) \cap T^{d}$ has positive Lebesgue measure on $T^{d}$. Then $f$ is identically zero on $\Delta$.

Proof. First, let us assume that $\Delta$ is a polydisk in $\mathbb{C}^{d}$, that is, $\Delta$ is a product of $d$ disks in $\mathbb{C}$. We use an induction on the dimension $d$. When $d=1$, the assertion is 
trivial since $f^{-1}(0) \cap S^{1}$ is finite if $f$ is not identically zero. Suppose that, for $d \geq 2$, the assertion is true in the dimension $d-1$. Let $\Delta \subset \mathbb{C}^{d}$ be a polydisk and let $f$ be a holomorphic function on $\Delta$. Suppose that $f$ is not identically zero. We would like to show that $\nu_{d}\left(f^{-1}(0) \cap T^{d}\right)=0$. We may assume that $\Delta \cap T^{d} \neq \emptyset$. In the following, a point in $\mathbb{C}^{d}$ is written as $(z, w)$ with $z \in \mathbb{C}^{d-1}$ and $w \in \mathbb{C}$. We set $\Delta=V_{d-1} \times V_{1}$ where $V_{d-1} \subset \mathbb{C}^{d-1}$ is a polydisk and $V_{1} \subset \mathbb{C}$ is a disk. For any $(z, w) \in \Delta$, we set $g_{w}(z)=h_{z}(w)=f(z, w)$. The functions $g_{w}$ and $h_{z}$ are holomorphic on $V_{d-1}$ and $V_{1}$, respectively. Fubini's theorem shows

$$
\nu_{d}\left(f^{-1}(0) \cap T^{d}\right)=\int_{S^{1}} \nu_{d-1}\left(g_{w}^{-1}(0) \cap T^{d-1}\right) d \nu_{1}(w) .
$$

We set $M=\left\{w \in S^{1} ; \nu_{d-1}\left(g_{w}^{-1}(0) \cap T^{d-1}\right)>0\right\}$. We show that $\nu_{1}(M)=0$. By induction hypothesis, $w \in M$ if and only if $g_{w}$ is identically zero on $V_{d-1}$. Suppose that $M \neq \emptyset$ and we take $w_{o} \in M$. Since $g_{w_{o}}=0$ on $V_{d-1}, h_{z}\left(w_{o}\right)=0$ for any $z \in V_{d-1}$. Since $f$ is not identically zero, one can take a point $z_{o} \in V_{d-1}$ such that $h_{z_{o}}$ is not identically zero on $V_{1}$. For such a point, one can take $z_{o}$ in $V_{d-1} \cap T^{d-1}$ by the induction hypothesis and the assumption that $f$ is not identically zero. Since $h_{z_{o}}$ is holomorphic on the disk $V_{1}$ in $\mathbb{C}, h_{z_{o}}^{-1}(0)$ is a discrete set containing $w_{o}$. There exists an open neighborhood $I$ of $w_{o}$ in $V_{1}$ such that $h_{z_{o}}^{-1}(0) \cap I=\left\{w_{o}\right\}$. We clearly have $M \cap I=\left\{w_{o}\right\}$. This means that $M$ is a discrete set in $S^{1} \cap V_{1}$ and hence $M$ is at most countable and its Lebesgue measure is zero. Now, let $\Delta \subset \mathbb{C}^{d}$ be a general domain and let $f$ be a holomorphic function on $\Delta$. Since $\Delta$ is second countable, one can find a countable open overing $\left\{\Delta_{n}\right\}_{n=1}^{\infty}$ of $\Delta$ such that each $\Delta_{n}$ is a polydisk. Suppose that $\nu_{d}\left(f^{-1}(0) \cap T^{d}\right)>0$. Then, we have

$$
0<\nu_{d}\left(f^{-1}(0) \cap T^{d}\right) \leq \sum_{n} \nu_{d}\left(\Delta_{n} \cap f^{-1}(0) \cap T^{d}\right),
$$

and hence there exists an integer $n$ such that $\nu_{d}\left(\Delta_{n} \cap f^{-1}(0) \cap T^{d}\right)>0$. Thus, from what we have just proved above, $f$ is identically zero on $\Delta_{n}$. Now, the identity theorem for holomorphic functions shows that $f$ is identically zero on $\Delta$.

Lemma 4.2. Suppose that $U$ has an eigenvalue $\lambda$. Then $\lambda$ is an eigenvalue of $\widehat{U}(z)$ for any $z \in T_{\mathbb{C}}^{d}$.

Proof. Let $0 \neq w \in \mathcal{H}$ be an eigenvector of $\mathcal{U}$ with the eigenvalue $\lambda$. Then, there exists a Borel set $W \subset T^{d}$ such that $\nu_{d}(W)=1$ and $\widehat{U}(z) w(z)=\lambda w(z)$ for all $z \in W$. We may set $w(z)=0$ for $z \in T^{d} \backslash W$. We have $\chi_{\lambda}(z)=0$ for any $z \in T^{d} \backslash w^{-1}(0)$, where $\chi_{\lambda}(z):=\chi(\lambda, z)$ is a Laurent polynomial in $z \in T_{\mathbb{C}}^{d}$. Since $w \neq 0$, we have $\nu_{d}\left(T^{d} \backslash w^{-1}(0)\right)>0$. Therefore, Lemma 4.1 shows that the function $\chi_{\lambda}$ is identically zero on $T_{\mathbb{C}}^{d}$. Thus, $\lambda$ is an eigenvalue of $\widehat{U}(z)$ for any $z \in T_{\mathbb{C}}^{d}$.

We have to show the converse to Lemma 4.2. Suppose that $\lambda \in S^{1}$ is an eigenvalue of the unitary matrix $\widehat{U}(z)$ for any $z \in T^{d}$. By Lemma 4.1, we have $\chi(\lambda, z)=0$ for any $z \in T_{\mathbb{C}}^{d}$. Therefore, for each fixed $z \in T_{\mathbb{C}}^{d}$, the polynomial $\chi(\zeta, z)$ in $\zeta \in \mathbb{C}$ 
is divisible by $\zeta-\lambda$. However, the multiplicity of $\zeta-\lambda$ in $\chi(\zeta, z)$ depends on $z \in T_{\mathbb{C}}^{d}$. To handle this situation, let $R_{d}$ denote the ring of Laurent polynomials in $z=\left(z_{1}, \ldots, z_{d}\right)$ with complex coefficients. The quotient field of $R_{d}$, denoted by $k_{d}$, is the field of rational functions on $\mathbb{C}^{d}$. Let $R_{d}[\zeta]$, respectively $k_{d}[\zeta]$, be the ring of polynomials in one variable $\zeta$ with coefficients in $R_{d}$, respectively, in $k_{d}$. We have $\chi \in R_{d}[\zeta] \subset k_{d}[\zeta]$. Since $\chi(\lambda, z)=0$ for any $z \in T_{\mathbb{C}}^{d}$, the polynomial $\zeta-\lambda \in k_{d}[\zeta]$ divides $\chi$ in $k_{d}[\zeta]$.

Lemma 4.3. Let $p, q \in k_{d}[\zeta]$ be monic polynomials and let $r \in R_{d}[\zeta]$. If $r=p q$, then $p, q \in R_{d}[\zeta]$.

Proof. It follows from Lemma 2 in Supplement of Ref. 4 that each coefficient of $p$ and $q$ is holomorphic on $T_{\mathbb{C}}^{d}$. Thus, what we need is to prove that a rational function $m(z)$ in $z \in \mathbb{C}^{d}$ which is holomorphic on $T_{\mathbb{C}}^{d}$ is actually a Laurent polynomial in $z$. We use an induction on $d$. Let us consider the case where $d=1$. Let $g / f(f, g \in \mathbb{C}[z])$ be a rational function holomorphic on $T_{\mathbb{C}}^{1}$. We may suppose that $f$ and $g$ have no common roots. Since $g / f$ is holomorphic on $T_{\mathbb{C}}^{1}$, it is possible for $f$ to have a root only at the origin. Thus, $f$ is a monomial and hence $g / f$ is a Laurent polynomial.

Suppose that the assertion holds in the dimension $d-1$. For $z \in \mathbb{C}^{d}$, we write, as before, $z=(\xi, w)$ with $\xi \in \mathbb{C}$ and $w=\left(w_{1}, \ldots, w_{d-1}\right) \in \mathbb{C}^{d-1}$. Let $m(\xi, w)$ be a rational function in $(\xi, w) \in \mathbb{C}^{d}$ holomorphic in $T_{\mathbb{C}}^{d}$. We write $m(\xi, w)=$ $g(\xi, w) / f(\xi, w)$ with polynomials $f$ and $g$ having no common irreducible factors in $\mathbb{C}[\xi, w]$. The assertion is trivial if $f$ is constant. Thus, suppose that $f$ is non-constant. First, suppose that $g$ is a nonzero constant. Since $m=g / f$ is holomorphic on $T_{\mathbb{C}}^{d}$, $f$ can not be zero on $T_{\mathbb{C}}^{d}$. Let $s=\xi w_{1} \cdots w_{d-1}$. By Hilbert's Nullstelensatz (see, for instance, Chap. 4 of Ref. 6), each irreducible factor of $f$ divides $s$, and hence $f$ is a monomial. Next, suppose that $g$ is not constant. We may suppose that $g$ has a positive degree in $\xi$, and we write $g=b_{0} \xi^{m}+\cdots+b_{m}$ with $m>0, b_{j} \in \mathbb{C}[w]$ and $b_{0} \neq 0$. If $f$ is constant in $\xi$, then $f \in \mathbb{C}[w]$, and $m=\left(b_{0} / f\right) \xi^{m}+\cdots+\left(b_{m} / f\right)$. Since $b_{j} / f$ 's are rational functions on $\mathbb{C}^{d-1}$ holomorphic in $T_{\mathbb{C}}^{d-1}$, the inductive hypothesis shows that these are Laurent polynomials in $w$. Thus, we may suppose that $f$ and $g$ have positive degrees in $\xi$. Let $f$ be a polynomial in $\xi$ of degree $l>0$, and we write $f=a_{0} \xi^{l}+\cdots+a_{l}$ with $a_{j} \in \mathbb{C}[w]$ and $a_{0} \neq 0$. Let $r \in \mathbb{C}[w]$ be the resultant of the polynomials $f, g$ with respect to $\xi$. (See, for instance, Chap. 3 of Ref. 6 for the properties of resultants.) Since $f$ and $g$ have no common factors, $r$ is not the zero polynomial. Suppose that $a_{l} \neq 0$. We set $a=a_{l} r \in \mathbb{C}[w]$. Then, the polynomial $a$ is not the zero polynomial and $U=\left\{w \in \mathbb{C}^{d-1} ; a(w) \neq 0\right\}$ is an open dense subset in $\mathbb{C}^{d-1}$. Fix $w_{o} \in U \cap T_{\mathbb{C}}^{d-1}$. Since $r\left(w_{o}\right) \neq 0, f\left(\xi, w_{o}\right)$ and $g\left(\xi, w_{o}\right)$ have no common zeros as polynomials in $\xi \in \mathbb{C}$. Since $m=g / f$ is holomorphic on $T_{\mathbb{C}}^{d}, f\left(\xi, w_{o}\right)=0$ only at $\xi=0$. However, since $a_{l}\left(w_{o}\right) \neq 0$, we have $f\left(\xi, w_{o}\right) \neq 0$ for any $\xi \in \mathbb{C}$, which is a contradiction to the assumption that $f$ has a positive degree in $\xi$. Thus, $a_{l}$ is zero. This shows that $f$ is divisible by $\xi$. We write $f=\xi^{k} h$ where $k>0$ and $h$ is a polynomial in $\mathbb{C}[\xi, w]$ which is not divisible by $\xi$. Then $\xi^{k} m=g / h$ is again a 
rational function holomorphic on $T_{\mathbb{C}}^{d}$. Applying the above discussion shows that $h$ must be a constant in $\xi$. Thus, $h \in \mathbb{C}[w]$. Therefore, the previous discussion shows that $\xi^{k} m=g(\xi, w) / h(w)$ is a Laurent polynomial in $(\xi, w)$.

Proof of Theorem 1.3. (1) Suppose, as above, that $\lambda \in S^{1}$ is an eigenvalue of $\widehat{U}(z)$ for any $z \in T^{d}$ and hence for any $z \in T_{\mathbb{C}}^{d}$. We divide $\chi$ by $\zeta-\lambda$ in $k_{d}[\zeta]$ and write $\chi(\zeta, z)=(\zeta-\lambda)^{m} q(\zeta, z)$, where $q$ is not divisible by $\zeta-\lambda$ in $k_{d}[\zeta]$. By Lemma 4.3, $q$ is in $R_{d}[\zeta]$. By the Hamilton-Cayley theorem, we have

$$
0=\chi(\widehat{U}(z), z)=(\widehat{U}(z)-\lambda)^{m} q(\widehat{U}(z), z)
$$

as a matrix for any $z \in T_{\mathbb{C}}^{d}$. We note that $q(\widehat{U}(z), z)$ is not identically zero on $T^{d}$. Indeed, suppose contrary that $q(\widehat{U}(z), z)=0$ for any $z \in T^{d}$. Then, for any fixed $z \in T^{d}, q(\zeta, z)$ is divisible by the minimal polynomial of the matrix $\widehat{U}(z)$. Since $\widehat{U}(z)$ has $\lambda$ as an eigenvalue, $q(\zeta, z)$ is divisible by $\zeta-\lambda$ for any $z \in T^{d}$. Hence $q(\lambda, z)=0$ for any $z \in T_{\mathbb{C}}^{d}$ by Lemma 4.1. Thus, $q$ is divisible by $\zeta-\lambda$ in $k_{d}[\zeta]$ which is a contradiction. Therefore, $q(\widehat{U}(z), z)$ is not identically zero. By the Fourier transform, the bounded operator $q(U, \tau)$ on $H$ is not identically zero. From 4.1), it follows that $0=\chi(U, \tau)=(U-\lambda)^{m} q(U, \tau)$. We take $w \in H$ such that $h:=q(U, \tau) w$ is not zero. Since $(U-\lambda)^{m} h=0$, there exists a number $j(1 \leq j<m)$ such that $(U-\lambda)^{j} h \neq 0$ and $(U-\lambda)^{j+1} h=0$. Then $v=(U-\lambda)^{j} h$ is an eigenvector of $U$ with the eigenvalue $\lambda$.

To close this section, it would be worth mentioning that the proof given above also shows the following proposition.

Proposition 4.4. Let $\lambda$ be an eigenvalue of the periodic unitary transition operator $U$ on $H=\ell^{2}\left(\mathbb{Z}^{d}, W\right)$. We decompose the characteristic polynomial $\chi(\zeta, z)$ in $k_{d}[\zeta]$ in the form $\chi(\zeta, z)=(\zeta-\lambda)^{m} q(\zeta, z)$, where $q(\zeta, z) \in R_{d}[\zeta]$ is an irreducible polynomial in $R_{d}[\zeta]$. Then, for any $w \in \ell^{2}\left(\mathbb{Z}^{d}, W\right)$, we have $q(U, \tau) w=0$ or $(U-\lambda)^{j} q(U, \tau) w$ is an eigenfunction of $U$ for some $j$ with $1 \leq j<m$.

\section{Absolute Continuity of the Continuous Spectrum}

Finally, we discuss the absolute continuity of the continuous spectrum of a periodic unitary transition operator $U$. As before, let $\operatorname{spec}(U)_{p}=\left\{\omega_{1}, \ldots, \omega_{K}\right\}$ be the set of eigenvalues of $U$ and let $\pi_{j}$ be the orthogonal projection onto the eigenspace of $U$ corresponding to $\omega_{j}$. We set $\pi_{p}=\pi_{1}+\cdots+\pi_{K}$ and $\pi_{c}=I-\pi_{p}$. If $\operatorname{spec}(U)_{p}=\emptyset$, then we set $\pi_{p}=0$. The characteristic polynomial $\chi$ defined in (3.2) is divisible by $\zeta-\omega_{j}$ in the ring of polynomials $k_{d}[\zeta]$ with coefficients in the field $k_{d}$ of rational functions in $z=\left(z_{1}, \ldots, z_{d}\right)$. The characteristic polynomial $\chi$ is monic in the sense that the coefficient of the leading term of $\chi$ is the identity in $k_{d}$. The ring $k_{d}[\zeta]$ is a unique factorization domain (UFD for short), and hence $\chi \in k_{d}[\zeta]$ can be decomposed into powers of monic irreducible elements in $k_{d}[\zeta]$. It should be remarked that the ring $R_{d}$ of Laurent polynomials in $z=\left(z_{1}, \ldots, z_{d}\right)$ is a UFD because it 
is a localization of the ring $\mathbb{C}\left[z_{1}, \ldots, z_{d}\right]$ of polynomials in $z$. Thus, $R_{d}[\zeta]$ is also a UFD, and hence $\chi$ can be decomposed into irreducibles in $R_{d}[\zeta]$. However, we need to use some properties of the notion of discriminants which are valid for polynomials with coefficients in a field. Since the polynomials $\zeta-\omega_{j}$ are irreducible, the decomposition of $\chi$ into monic irreducible elements in $k_{d}[\zeta]$ can be written as

$$
\chi(\zeta, z)=\prod_{j=1}^{K}\left(\zeta-\omega_{j}\right)^{m_{j}} \times \prod_{\rho=1}^{r} \pi_{\rho}(\zeta, z)^{n_{\rho}},
$$

where $\pi_{\rho} \in k_{d}[\zeta]$ are monic irreducible polynomials in $k_{d}[\zeta]$, and $\zeta-\omega_{j}(j=$ $1, \ldots, K)$ and $\pi_{\rho}(\rho=1, \ldots, r)$ are mutually different. We set

$$
\pi(\zeta, z)=\prod_{j=1}^{K}\left(\zeta-\omega_{j}\right) \times \prod_{\rho=1}^{r} \pi_{\rho}(\zeta, z)
$$

so that $\chi^{-1}(0)=\pi^{-1}(0) \subset T_{\mathbb{C}}^{d+1}$. If $\pi_{\rho}(1 \leq \rho \leq r)$ is of the form $\zeta-\lambda$ with a constant $\lambda \in \mathbb{C}$, then $\chi(\lambda, z)=0$ for any $z \in T_{\mathbb{C}}^{d}$ and hence by Theorem 1.3 (1), $\lambda$ is an eigenvalue of the operator $U$. Thus, $\lambda=\omega_{j}$ for some $j=1, \ldots, K$. However, $\pi_{\rho}$ is different from $\zeta-\omega_{j}$, and hence it is a contradiction. Thus, $\pi_{\rho}$ is not of the form $\zeta-\lambda$ with a constant $\lambda$. By Lemma 4.3, each polynomial $\pi_{\rho}$ in (5.2) is in $R_{d}[\zeta]$. Let $d_{\pi}$ denote the discriminant of $\pi$, namely, $d_{\pi}= \pm$ resultant of $\pi$ and $\partial_{\zeta} \pi$. $d_{\pi}$ is a Laurent polynomial in $z \in T_{\mathbb{C}}^{d}$. Note that $d_{\pi}$ is identically zero if and only if $\pi$ and $\partial_{\zeta} \pi$ has a common irreducible factor in $k_{d}[\zeta]$. Since $\pi$ is a product of mutually different irreducible elements in $k_{d}[\zeta], d_{\pi}$ is not identically zero. Hence the discriminant set $D=d_{\pi}^{-1}(0) \subset T_{\mathbb{C}}^{d}$ is an algebraic variety such that $T_{\mathbb{C}}^{d} \backslash D$ is a connected open dense set (Ref. 으, Chap. 1). By Lemma 4.1, $T^{d} \cap D$ is a closed set in $T^{d}$ having Lebesgue measure zero. For any $z \in T_{\mathbb{C}}^{d} \backslash D$, the equation $\pi(\zeta, z)=0$ has $K+L=\operatorname{deg} \pi$ distinct roots in $\mathbb{C}$ because $d_{\pi}=d_{\pi}(z)$ is the discriminant of $\pi(\zeta, z)$ in $\mathbb{C}[\zeta]$ when $z$ is fixed and hence $\pi(\zeta, z)$ does not have multiple roots for fixed $z \notin D$. (see Ref. $[6$ for the properties of discriminants.)

Lemma 5.1. Let $z_{o} \in T_{\mathbb{C}}^{d} \backslash D$. Then, there exists a neighborhood $V \subset T_{\mathbb{C}}^{d} \backslash D$ of $z_{o}$ and holomorphic functions $\lambda_{1}(z), \ldots, \lambda_{L}(z)$ on $V$ such that, for each $z \in V$, the roots of the equation $\pi(\zeta, z)=0$ is given by $\omega_{1}, \ldots, \omega_{K}, \lambda_{1}(z), \ldots, \lambda_{L}(z)$.

Proof. Let $\omega_{1}, \ldots, \omega_{K}, \lambda_{1}, \ldots, \lambda_{L}$ be the roots of the equation $\pi\left(\zeta, z_{o}\right)=0$, each of which is different from others. Let $\lambda_{o}$ denote one of $\lambda_{j}$ 's. Since $z_{o} \notin D$ and since $\pi\left(\lambda_{o}, z_{o}\right)=0$, we have $\partial_{\zeta} \pi\left(\lambda_{o}, z_{o}\right) \neq 0$. Applying the implicit function theorem (Ref. [8. Chap. 1), there exists a neighborhood $V$ of $z_{o}$ and a holomorphic function $\lambda(z)$ on $V$ such that $\pi(\lambda(z), z)=0$ for any $z \in V$ and $\lambda\left(z_{o}\right)=\lambda_{o}$. This shows the lemma.

The projections onto algebraic (generalized) eigenspaces (called the eigenprojection in Ref. 15) are also holomorphic near $z_{o} \in T^{d} \backslash D$. Namely, we have the following lemma. 
Lemma 5.2. Let $z_{o} \in T_{\mathbb{C}}^{d} \backslash D$. Let $V_{o}$ be an open neighborhood of $z_{o}$ in $T_{\mathbb{C}}^{d} \backslash D$ as in Lemma 5.1. Then there is an open neighborhood $V_{1}$ of $z_{o}$ in $V_{o}$ and holomorphic projection-valued functions $R_{j}(z)(1 \leq j \leq K), P_{k}(z)(1 \leq k \leq L)$ such that $R_{j}(z)$, $P_{k}(z)$ are the projections onto the algebraic eigenspaces corresponding to $\omega_{j}, \lambda_{k}(z)$, respectively, for each $z \in V_{1}$.

Proof. As before, let $\omega_{1}, \ldots, \omega_{K}, \lambda_{1}, \ldots, \lambda_{L}$ be mutually different eigenvalues of the matrix $\widehat{U}\left(z_{o}\right)$. Let $\lambda_{o}$ be one of them. We take a small circle $\Gamma$ around $\lambda_{o}$ such that $\Gamma$ and the disk bounded by $\Gamma$ do not contain any other eigenvalues. Let $\lambda_{o}(z)$ denote the eigenvalue of $\widehat{U}(z)$ in $V_{o}$ such that $\lambda_{o}\left(z_{o}\right)=\lambda_{o}$ which is holomorphic on $V_{o}$, and let $\lambda(z)$ be any other holomorphic function which is an eigenvalue of $\widehat{U}(z)$ in $V_{o}$. Since $\lambda_{o}(z)$ and $\lambda(z)$ are continuous, there exists a neighborhood $V_{1}$ of $z_{o}$ in $V_{o}$ such that, for each $z \in V_{1}, \lambda(z)$ is not contained in $\Gamma$ and the disk bounded by $\Gamma$ and $\lambda_{o}(z)$ is inside $\Gamma$. Then, the eigenprojection $P(z)$ onto the algebraic eigenspace corresponding to $\lambda_{o}(z)$ along other algebraic eigenspaces is given by the contour integral (Ref. 15 Chap. 1)

$$
P(z)=\frac{1}{2 \pi i} \int_{\Gamma}(\zeta-\widehat{U}(z))^{-1} d \zeta
$$

From this expression, $P(z)$ is holomorphic in $z \in V_{1}$.

We remark that, when $z_{o} \in T^{d} \backslash D, R_{j}(z)$ and $P_{k}(z)$ in Lemma 5.2 are smooth functions on $T^{d} \cap V_{1}$ which are the orthogonal projections onto the geometric eigenspaces because $\widehat{U}(z)$ is a unitary matrix for $z \in T^{d}$. For $R_{j}(z)$, we have the following lemma.

Lemma 5.3. In the above, $R_{j}(z)(1 \leq j \leq K)$ is extended to $T^{d} \backslash D$ as a smooth orthogonal projection-valued bounded function, and it is the orthogonal projection onto the eigenspace corresponding to the eigenvalue $\omega_{j}$ of $\widehat{U}(z)$ for each $z \in T^{d} \backslash D$.

Proof. For each fixed $z \in T_{\mathbb{C}}^{d}$, let $R_{j}(z)$ be the eigenprojection onto the algebraic eigenspace corresponding to the eigenvalue $\omega_{j}$ of $\widehat{U}(z)$ defined by a contour integral as in (5.3). $R_{j}(z)$ is well-defined as a single-valued function on $T_{\mathbb{C}}^{d}$. By Lemma [5.2, it is holomorphic on $T_{\mathbb{C}}^{d} \backslash D$, and hence its restriction to $T^{d} \backslash D$ is smooth. For $z \in T^{d}$, $\widehat{U}(z)$ is a unitary matrix and hence $R_{j}(z)$ is the orthogonal projection onto the (geometric) eigenspaces corresponding to $\omega_{j}$. Thus, the operator norm of $R_{j}(z)$ for $z \in T^{d}$ is bounded.

Lemma 5.4. Let $R_{j}(z)$ be the orthogonal projection-valued functions on $T^{d} \backslash D$ in Lemma 5.3. Then, the orthogonal projection $\pi_{j}$ onto the eigenspace of $\mathcal{U}$ corresponding to the eigenvalue $\omega_{j}$ is given by the following formula:

$$
\left(\pi_{j} w\right)(z)=R_{j}(z) w(z) \quad\left(w \in L^{2}\left(T^{d}, \mathbb{C}^{D}\right), z \in T^{d} \backslash D\right) .
$$

Proof. Since $T^{d} \cap D$ has Lebesgue measure zero and since $R_{j}(z)$ is bounded on $T^{d} \backslash D$, the right-hand side of the above expression defines a bounded operator $R_{j}$ on 
$L^{2}\left(T^{d}, \mathbb{C}^{D}\right)$ which commutes with $\mathcal{U}$. By Lemma 5.3, the image of $R_{j}$ is contained in that of $\pi_{j}$. But, it is obvious that, for $w \in \operatorname{Im}\left(\pi_{j}\right), w(z)$ is an eigenvector of $\widehat{U}(z)$ with eigenvalue $\omega_{j}$ when $w(z) \neq 0$. Thus, we have $R_{j} w=w$, which completes the proof.

For each $z_{o} \in T^{d} \backslash D$, we take a small neighborhood $V_{z_{o}}$ such that $\overline{V_{z_{o}}}$ is a compact subset contained in a neighborhood of $z_{0}$ as in Lemmas 5.1 and 5.2. We set $W_{z_{o}}=V_{z_{o}} \cap T^{d}$. We may assume that $W_{z_{o}}$ is connected. Let $\mathcal{H}\left(W_{z_{o}}\right)$ be the closed subspace in $\mathcal{H}$ consisting of all $w \in L^{2}\left(T^{d}, \mathbb{C}^{D}\right)$ whose essential support is contained in $W_{z_{o}}$. It is obvious that the orthogonal projection onto $\mathcal{H}\left(W_{z_{o}}\right)$ is given by the multiplication of the characteristic function of $W_{z_{o}}$, and hence it commutes with $\mathcal{U}$. Thus, the spectral projection of $\mathcal{U}$ also commutes with the orthogonal projection onto $\mathcal{H}\left(W_{z_{o}}\right)$. Let $R_{j}(z), P_{i}(z)(1 \leq j \leq K, 1 \leq i \leq L)$ denote the projection-valued holomorphic function on $V_{z_{o}}$ given in Lemma [5.2, As in Ref. 15], we have

$$
\sum_{j=1}^{K} R_{j}(z)+\sum_{i=1}^{L} P_{i}(z)=I \quad\left(z \in V_{z_{o}}\right) .
$$

By Lemma [5.4 for each $w \in \mathcal{H}\left(W_{z_{o}}\right)$, we see

$$
w(z)=\left(\pi_{p} w\right)(z)+\sum_{i=1}^{L} P_{i}(z) w(z)
$$

for almost all $z \in W_{z_{o}}$. Since $w=\pi_{p} w+\pi_{c} w$, we have

$$
\left(\pi_{c} w\right)(z)=\sum_{i=1}^{L} P_{i}(z) w(z) \quad\left(w \in \mathcal{H}\left(W_{z_{o}}\right)\right) .
$$

From (5.4), we obtain

$$
\mathcal{U}^{n} \pi_{c} w(z)=\sum_{i=1}^{L} \lambda_{i}(z)^{n} P_{i}(z) w(z) \quad\left(w \in \mathcal{H}\left(W_{z_{o}}\right)\right)
$$

for any integer $n$. Since the ring of Laurent polynomials on $S^{1}$ is dense in the space $C\left(S^{1}\right)$ of continuous functions on $S^{1}$ with respect to the supremum norm, we see

$$
f(\mathcal{U}) \pi_{c} w(z)=\sum_{i=1}^{L} f\left(\lambda_{i}(z)\right) P_{i}(z) w(z) \quad\left(w \in \mathcal{H}\left(W_{z_{o}}\right)\right)
$$

for any $f \in C\left(S^{1}\right)$.

Proposition 5.5. Let $V_{z_{o}} \subset T_{\mathbb{C}^{D}}^{d} \backslash D$ and $W_{z_{o}} \subset T^{d} \backslash D$ be as above. Let $w \in$ $\mathcal{H}\left(W_{z_{o}}\right)$. Then, the spectral measure $\left\|E(\cdot) \pi_{c} w\right\|^{2}$ of $\pi_{c} w$ is absolutely continuous with respect to the Lebesgue measure.

Proof. We first show that the gradient $\nabla \lambda_{k}$ of the eigenvalues $\lambda_{k}(z)(1 \leq k \leq L)$, considered as a function on $W_{z_{o}}$, does not vanish almost everywhere on $W_{z_{o}}$. To 
show this, let $\left(\theta_{1}, \ldots, \theta_{d}\right) \in \mathbb{R}^{d}$ be a coordinates on $W_{z_{o}}$ defined so that $z=$ $\left(e^{i \theta_{1}}, \ldots, e^{i \theta_{d}}\right) \in W_{z_{o}}$. We denote $\partial_{\theta_{j}}$ the partial derivative with respect to $\theta_{j}$. Then $\partial_{\theta_{j}} \lambda_{k}=i z_{j} \partial_{j} \lambda_{k}$ on $W_{z_{o}}$, where $\partial_{j} \lambda_{k}$ denotes the derivative in $z_{j}$ of the holomorphic function $\lambda_{k}$. Let $N_{k} \subset W_{z_{o}}$ be the set of points $z \in W_{z_{o}}$ where $\nabla \lambda_{k}(z)=0$. Thus, $N_{k}$ is the intersection of the sets $\left(\partial_{j} \lambda_{k}\right)^{-1}(0) \cap T^{d}$ for $j=1, \ldots, d$. If $N_{k}$ has positive Lebesgue measure on $T^{d}$, then by Lemma 4.1, $\partial_{j} \lambda_{k}$ vanishes identically on $V_{z_{o}}$ for any $j=1, \ldots, d$. Therefore, $\lambda_{k}$ is constant on $V_{z_{o}}$. Now, we have $\pi\left(\lambda_{k}, z\right)=0$ in $z \in V_{z_{o}}$. By the identity theorem for holomorphic functions, $\pi\left(\lambda_{k}, z\right)=0$ for any $z \in T_{\mathbb{C}}^{d}$. This means that the irreducible monic polynomial $\zeta-\lambda_{k} \in k_{d}[\zeta]$ divides $\pi$, which is a contradiction to the fact that each $\pi_{\rho}$ in (5.2) is not of the form $\zeta-\lambda$. Therefore, $\nabla \lambda_{k}$ does not vanish almost everywhere on $W_{z_{o}}$.

Now, let $f$ be a continuous function on $S^{1}$. Since $P_{i}(z)$ and $P_{j}(z)(i \neq j)$ are orthogonal to each other for $z \in W_{z_{o}}$, (5.6) shows

$$
\left\|f(\mathcal{U}) \pi_{c} w\right\|^{2}=\sum_{i=1}^{L} \int_{W_{z_{o}}}\left|f\left(\lambda_{i}(z)\right)\right|^{2}\left\|P_{i}(z) w(z)\right\|_{\mathbb{C}^{D}}^{2} d \nu_{d}(z)
$$

As is shown in the above, we have $\nu_{d}\left(W_{z_{o}}\right)=\nu_{d}\left(W_{z_{o}} \cap N_{i}^{c}\right)$ where $N_{i}^{c}$ is the complement of $N_{i}$. The function $\lambda_{i}$ restricted to $W_{z_{o}} \cap N_{i}^{c}$ has no critical points, and hence for each $t \in S^{1}$, the set $\lambda_{i}^{-1}(t) \cap W_{z_{o}} \cap N_{i}^{c}$ is a smooth hyper-surface. For each $t \in S^{1}$ and $i=1, \ldots, K$, we set

$$
\Gamma_{i}(t)=\int_{\lambda_{i}^{-1}(t) \cap W_{z_{o}} \cap N_{i}^{c}}\left\|P_{i}(z) w(z)\right\|_{\mathbb{C}^{D}}^{2} \frac{d S_{t}(z)}{\left|\nabla \lambda_{i}(z)\right|}
$$

if $\lambda_{i}^{-1}(t) \cap W_{z_{o}} \cap N_{i}^{c} \neq \emptyset$, where $d S_{t}$ denotes the volume element of the hypersurface $\lambda_{i}^{-1}(t) \cap W_{z_{o}} \cap N_{i}^{c}$. Then the coarea formula (see Ref. 5 or Ref. 14, Appendix A) shows that $\Gamma_{i}(t)$ is an $L^{1}$-function on $S^{1}$ and

$\int_{S^{1}}|f(t)|^{2} d\left\|E(t) \pi_{c} w\right\|^{2}=\left\|f(\mathcal{U}) \pi_{c} w\right\|^{2}=\int_{S^{1}}|f(t)|^{2} \Gamma(t) d \nu_{1}(t), \quad \Gamma(t)=\sum_{i=1}^{L} \Gamma_{i}(t)$,

which proves that the measure $d\left\|E(t) \pi_{c} w\right\|^{2}$ is absolutely continuous with respect to $d \nu_{1}(t)$.

Proof of Theorem 1.3(2). For each $z_{o} \in T^{d} \backslash D$, we take a small neighborhood $V_{z_{o}}$ such that $\overline{V_{z_{o}}}$ is a compact set contained in a neighborhood of $z_{o}$ as in Lemmas 5.1 and 5.2. We set, as before, $W_{z_{o}}=T^{d} \cap V_{z_{o}}$. Then $\left\{W_{z_{o}}\right\}_{z_{o} \in T^{d} \backslash D}$ is an open covering of $T^{d} \backslash D$. We can choose a locally finite countable open covering $\left\{W_{i}\right\}_{i=1}^{\infty}$ which is a refinement of a countable sub-covering of the covering $\left\{W_{z_{o}}\right\}_{z_{o} \in T^{d} \backslash D}$. We take a partition of unity $\left\{\rho_{i}\right\}_{i=1}^{\infty}$ subordinated to the covering $\left\{W_{i}\right\}_{i=1}^{\infty}$. Then, the sum $\sum_{i=1}^{\infty} M_{i}$ converges in the strong operator topology and equals the identity operator on $\mathcal{H}$. Let $w \in \mathcal{H}$. Let $\Lambda \subset S^{1}$ be a Borel subset having Lebesgue measure zero. Since $\mathcal{U}$ commutes with the operator $M_{i}$ defined by the multiplication by $\rho_{i}$, 
the spectral measure $E(\Lambda)$ and the projection $\pi_{c}$ also commute with $M_{i}$. Therefore, we obtain

$$
\left\langle E(\Lambda) \pi_{c} w, \pi_{c} w\right\rangle=\sum_{i=1}^{\infty} \int_{T^{d} \backslash D}\left\|E(\Lambda) \pi_{c}\left(\rho_{i}^{1 / 2} w\right)(z)\right\|_{\mathbb{C}^{D}}^{2} d \nu_{d}(z) .
$$

Now Proposition 5.5 shows that $E(\Lambda) \pi_{c}\left(\rho_{i}^{1 / 2} w\right)=0$ in $\mathcal{H}$. Therefore, we have $E(\Lambda) \pi_{c} w=0$, which completes the proof of Theorem 1.3 (2).

\section{Acknowledgments}

The author would like to thank professor Alain Joye for his comments about the Wiener formula, professor Yusuke Higuchi for the comments on the work ${ }^{10}$ where a lemma essentially the same as our Lemma 4.1 below is used in a similar purpose, and professor Serge Richard for stimulate discussion.

The author is partially supported by JSPS KAKENHI Grant Nos. 25400068, 18K03267, 24340031, 15H02055 and 17H06465.

\section{References}

1. D. Aharonov, A. Ambainis, J. Kempe and U. Vazirani, Quantum walks on graphs, Proc. of the 33rd Annual ACM Symp. on Theory of Computing (Hersonissos, Greece, ACM, New York, USA, 2001), pp. 55-59.

2. Y. Aharonov, L. Davidovich and N. Zagury, Quantum random walks, Phys. Rev. A 48 (1993) 1687-1690.

3. A. Ambainis, E. Bach, A. Nayak, A. Vishwanath and J. Watrous, One-dimensional quantum walks, Proc. of the 33rd Annual ACM Symp. on Theory of Computing (2001), pp. 37-49.

4. H. Baumgärtel, Analytic Perturbation Theory for Matrices and Operators, Operator Theory: Advances and Applications, Vol. 15 (Birkhäuser Verlag, 1985).

5. I. Chavel, Eigenvalues in Riemannian Geometry, Pure and Appl. Mathematics, Vol. 115 (Academic Press, Inc., Orlando, 1984).

6. D. Cox, J. Little and D. O'Shea, Ideals, Varieties, and Algorithms, 2nd. edn. (SpringerVerlag Inc., 1997).

7. C. Gérard and F. Nier, The Mourre theory for analytically fibered operators, J. Funct. Anal. 152 (1998) 202-219.

8. R. C. Gunning and H. Rossi, Analytic Functions of Several Complex Variables (Prentice-Hall, Inc., Englewood Cliffs, N.J., 1965).

9. Y. Higuchi, N. Konno, I. Sato and E. Segawa, Spectral and asymptotic properties of Grover walks on crystal lattices, J. Funct. Anal. 267 (2014) 4197-4235.

10. Y. Higuchi and Y. Nomura, Spectral structure of the Laplacian on a covering graph, Eur. J. Combin. 30 (2009) 570-585.

11. N. Inui, Y. Konishi and N. Konno, Localization of two-dimensional quantum walks, Phys. Rev. A 69 (2004) 052323.

12. N. Inui and N. Konno, Localization of multi-state quantum walk in one dimension, Physica A 353 (2005) 133-144.

13. N. Inui, N. Konno and E. Segawa, One-dimensional three-state quantum walk, Phys. Rev. E 72 (2005) 056112. 
14. J. Jost and X. Li-Jost, Calculus of Variations, Cambridge Studies in Advanced Mathematics, Vol. 64 (Cambridge Univ. Press, 1998).

15. T. Kato, Perturbation Theory for Liner Operators, Reprint of the 1980 edn. Classics in Mathematics (Springer-Verlag, 1995).

16. Y. Katznelson, An Introduction to Harmonic Analysis, 2nd edn. (Dover Publications, Inc., 1976).

17. N. Konno, A new type of limit theorems for the one-dimensional quantum random walk, J. Math. Soc. Japan 57 (2005) 1179-1195.

18. N. Konno, N. Obata and E. Segawa, Localization of the Grover walks on spidernets and free Meixner laws, Comm. Math. Phys. 322 (2013) 667-695.

19. M. Kotani and T. Sunada, Spectral geometry of crystal lattices, Contemp. Math. 338 (2003) 271-305.

20. A. Nayak and A. Vishwanath, Quantum walk on the line, preprint (2001), (arXiv: quant-ph/0010117).

21. M. Reed and B. Simon, Method of Modern Mathematical Physics, I (Functional analysis), Revised and Enlarged edn. (Academic Press, Inc., 1980).

22. T. Sunada, Topological Crystallography. With a View Towards Discrete Geometric Analysis, Surveys and Tutorials in the Applied Mathematical Sciences, Vol. 6 (Springer, 2013).

23. T. Sunada and T. Tata, Asymptotic behavior of quantum walks on the line, J. Funct. Anal. 262 (2012) 2608-2645.

24. K. Watabe, N. Kobayashi, M. Katori and N. Konno, Limit distributions of twodimensional quantum walks, Phys. Rev. A 77 (2008), Article ID: 062331, pp. 1-9.

25. J. Watrous, Quantum simulations of classical random walks and undirected graph connectivity, J. Comput. Syst. Sci. 62 (2001) 376-391. 